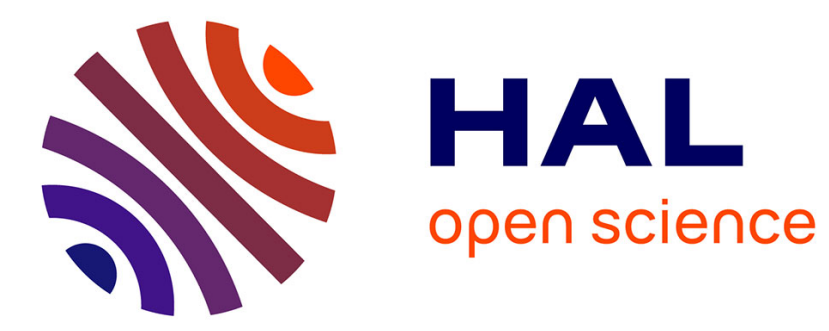

\title{
De la race comme un problème. Les sciences sociales et l'idée de nature
}

\author{
Fabrice Dhume
}

\section{To cite this version:}

Fabrice Dhume. De la race comme un problème. Les sciences sociales et l'idée de nature. Raison Présente, 2010, Racisme, race et sciences sociales, 174, p.53-65. halshs-01421985

\section{HAL Id: halshs-01421985 https://shs.hal.science/halshs-01421985}

Submitted on 2 Jan 2017

HAL is a multi-disciplinary open access archive for the deposit and dissemination of scientific research documents, whether they are published or not. The documents may come from teaching and research institutions in France or abroad, or from public or private research centers.
L'archive ouverte pluridisciplinaire HAL, est destinée au dépôt et à la diffusion de documents scientifiques de niveau recherche, publiés ou non, émanant des établissements d'enseignement et de recherche français ou étrangers, des laboratoires publics ou privés. 


\section{Dhume Fabrice, "Sur la notion de race comme un problème. Les sciences sociales et l'idée de nature ", Raison présente, n¹74 ("Racisme, race et sciences sociales $")$ ), p.53-65.}

\section{Sur la notion de race comme un problème. Les sciences sociales et l'idée de nature}

« Races " ${ }^{1}$ ? « Ethnies »? "C'est l'histoire qui dessine ces ensembles avant de les effacer ; il ne faut pas y chercher des faits biologiques bruts et définitifs qui, du fond de la "nature", s'imposeraient à l'histoire. ${ }^{2}$ Au premier abord, cette remarque de Michel Foucault traduit bien la perspective dite "constructiviste ». Les groupes et les catégories qui les désignent sont des produits historiques co-émergents et non-définitifs. Ils n'ont ni naturalité, ni essentialité, ni continuité a priori. La formule ouvre cependant à un second niveau de réflexion, qui porte cette fois sur la matrice intellectuelle de la « race » : la place de la nature dans notre conception du monde. II s'agit alors d'interroger le mouvement idéologique qui projette sur le réel un "fond de nature ", et ce faisant ramène ce dernier à un niveau supposé de primauté, de dureté, de fixité. La critique des sciences sociales s'est focalisée sur la confusion entre faits de nature et faits sociaux. On peut cependant se demander si la critique de l'idéologie raciste prend en charge la question de ce qu'est, " au fond ", la nature. Pour le dire autrement, si le constructivisme a permis de séparer des ordres, pour traiter de la naturalisation du social, ne s'est-il pas arrêté en chemin ?

En quoi parler de « race » signifie-t-il reprendre en charge ce que l'on a fait au nom de la race ? Pour le chercheur, cette question se présente comme un abîme, car la frontière qui sépare le champ scientifique de l'idéologico-politique semble ici bien incertaine. II y a des raisons à cela : on sait quelle a été la contribution des " sciences " à l'idéologie raciste, au $\mathrm{XIXe}$ et XXe siècles. Et l'on sait aussi que la déconstruction scientifico-politique de cette idéologie, après la seconde guerre mondiale, n'a cependant pas " dégagé " la recherche de la question de la responsabilité quant aux mots qu'elle utilise. Tout au contraire, peut-être. La notion de race a ceci de particulier qu'elle semble marquer un point d'articulation - et un point de fuite -, non seulement entre sciences, mais également entre science et politique et éthique, etc. Aussi le projet scientifique d'une autonomie à l'égard d'autres champs est-il mis à l'épreuve d'une catégorie qui ne cesse de poser le problème de passages incontrôlés entre des univers que l'on voudrait forclos. Ce sont ces frontières, ces passages, et leurs statuts

\footnotetext{
1 J'emploie le singulier italique - race - pour désigner un rapport social de domination par racisation, et le terme entre guillemets « race(s) » pour désigner la taxinomie.

2 FOUCAULT M., « Bio-histoire et bio-politique » (1976), Dits et écrits II, 1976-1988, Paris, Quarto Gallimard, 2001, p.97.
} 
que je voudrais ici questionner. Car, si les sciences sociales ont cherché à répondre à ce problème de frontières par l'analyse critique des passages en contrebande (ex : la racisation, définie comme "naturalisation du social »), cela semble s'être fait au prix d'une occultation du statut et de l'ordre de la nature. On a ainsi repoussé deux problèmes de l'horizon des sciences sociales : celui de la physicalité du social, et celui de l'unité sociale de la nature. ${ }^{3}$

Mon propos se veut une réflexion ouverte, avec ses incertitudes, son indécision. Indécision liée certes à l'objet, mais aussi à un choix de posture. Aussi la proposition du statut de problème indique-t-elle le maintien ouvert de la question. De ce fait, reconnaître la race comme un problème pourrait indiquer à la fois la singularité de cette question, et l'opportunité qu'elle nous donne d'interroger plus systématiquement le rapport des sciences sociales à leur objet.

\section{Sur l'idée de « race »}

L'idée de "race » offre une exceptionnelle mobilité intellectuelle, en articulant plusieurs réseaux de différenciation: physiques (les caractéristiques extérieures du corps), physiobiologiques (le fonctionnement interne du corps), moraux ou sociaux (ce que Buffon appelait le "naturel des différents peuples »), et politiques (au sens de la distribution des places dans l'ordre social). C'est à ce potentiel de connections que cette catégorie doit une partie de son succès. Elle facilite en effet des jugements et des classements intégrant simultanément plusieurs plans, des systèmes d'équivalences totalisants. C'est de ce syncrétisme, avec « la corrélation immédiate qu'il prétend établir entre du visible et de l'invisible $»^{4}$ que la catégorie tire sa puissance. Prenant appui sur la fondation naturaliste, l'idée de « race » apparaît en outre dotée d'une grande résistance : la force intrinsèque attribuée à la nature, pensée comme socle et matrice du vivant. Cette double dimension syncrétisme et naturalisme - donne à l'idée de " race » la force d'une évidence, difficile à entamer.

Face à cela, la critique scientifique de la seconde moitié du XXe siècle a surtout dénoncé la confusion du biologique et du social. La définition du racisme comme idéologie traduit l'idée que le recours à la «nature » pour déduire des compétences et des places n'est qu'un procédé de justification d'un système d'inégalités ${ }^{5}$. Le débat français (au moins) en sciences sociales semble globalement tenir pour acquis que l'idéo-logique du racisme tient dans la "biologisation de la pensée sociale, qui tente par ce biais de poser en absolu toute différence constatée ou supposée $»^{6}$, et ainsi de justifier un certain ordre social. A partir de

\footnotetext{
${ }^{3}$ Je rermercie Françoise Lorcerie pour ses remarques éclairantes. Mais ce texte n'engage que moi.

${ }^{4}$ OLENDER M., La chasse aux évidences. Sur quelques formes de racisme entre mythe et histoire, Paris, Galaade, 2005, p.14.

5 DE RUDDER V., « Les "races” sont inégales ou ne sont pas », Pluriel recherches, cahier n4, 1996.

${ }^{6}$ GUILLAUMIN C., L'idéologie raciste. Genèse et langage actuel, Paris, Gallimard, Folio essais, 2002, p.14.
} 
cette désubstantialisation, le débat se poursuit toutefois sur les usages de la notion de « race » dans le discours et l'analyse scientifiques. Faut-il l'utiliser ? Peut-on l'ignorer ? Et si l'on doit y recourir, à quel titre, de quelles manières et avec quelles précautions ? Ces questions ne cessent de hanter les sciences sociales, dans leur lien au politique - par exemple dans la polémique sur les "statistiques ethniques ». On comprend d'autant mieux ces débats en gardant à l'esprit que l'acceptation des catégories comme objets de travail suppose, au fond, leur admission relative comme outils de travail ; même lorsqu'on les manipule avec prudence, on ne peut séparer entièrement ces catégories de l'ordre de pensée qui leur confère leur sens.

\section{Des « races » aux « ethnies » : la ruse du transfert de nature en culture}

Ces débats font écho aux tentatives politico-scientifiques des années 1960, sous l'égide de I'UNESCO, « d'éliminer les races » en tant que catégories. Depuis, la contestation du racisme se fait au nom de la culture, dans un double sens : au sens d'objet de transmission éducative ${ }^{8}$ ou de médiation "interculturelle », d'une part, et comme catégorie intellectuelle opposée à une définition naturaliste des groupes, d'autre part. II est connu que la croyance dans la naturalité "des races" et dans la pertinence explicative de ces catégories se maintient pourtant, jusque dans certaines productions scientifiques ${ }^{9}$, malgré (ou plutôt avec) l'effort de conviction antiraciste. Le caractère problématique de l'idée de " race " se situe donc premièrement au niveau de la résistance de formes de catégorisations historiquement inventées, résistance découlant de leur institutionnalisation et de leur incorporation en tant que schème culturel. Les catégories de "races" et les formes racistes de jugement continuent à s'apprendre et se transmettre.

Face à cette impuissance à " changer les mentalités », le chercheur use de précautions. Par des tactiques sémantiques ou linguistiques ${ }^{10}$, il cherche à maintenir à distance un terme brûlant, corrosif, comme si le nom même de "race » accueillait nécessairement tous ses usages. Et ce, malgré le démenti de la linguistique ${ }^{11}$. Une autre ruse autorise à la fois la déculpabilisation et l'occultation du problème. II s'agit du remplacement des catégories « raciales » par celles " ethniques », le référent de culture se substituant à celui de nature. Cette stratégie n'a fait qu'infléchir la forme des usages par euphémisation : dire « ethnies » pour parler de "races" sans les nommer. Le "néo-racisme " emprunte désormais un référent culturaliste pour régénérer sa matrice inégalitariste dans des formes politiquement

\footnotetext{
${ }^{7}$ FICHET B., "Etranger et immigré, deux termes problématiques », Revue des sciences sociales de la France de l'Est, n' 20, 1993.

${ }^{8}$ RAPHAËL F., « La solution réside dans l'éducation », News d’Ill, n`81 (« L'Alsace côté ombre »), février 2005.

${ }^{9}$ N'oublions pas, derrière un débat « entre gens convaincus », que des scientifiques continuent leur œuvre de légitimation de la catégorie. Cf. FOUCART S., " La tentation de la race », Le Monde, 30.10.07.

${ }^{10}$ Par exemple : distinction entre racialisme ou racisme, utilisation avec ou sans guillemets, au singulier ou au pluriel, etc.

${ }^{11}$ BONNAFOUS S., FIALA P., « Est-ce que dire la race en présuppose l'existence ? », Mots, vol. 33, n¹, 1992.
} 
correctes. On tient alors la culture pour une seconde nature. Ce faisant, on confond aussi les ressources culturelles avec l'identité sociale - comme dans l'« interculturel ${ }^{12}$. Mais, en masquant la référence à la nature, ce transfert fait oublier que l'on a conservé un socle de raisonnement substantialiste, dans l'idée que l'humanité se fonde en nature. II y a donc là un second niveau de problème. La conception sous-jacente de la nature, présente autant dans les catégories raciales que leur critique, repose sur un présupposé ancien opposant nature et culture, et faisant de la première le socle de la seconde. En fin de compte, la critique des sciences sociales repose sur le degré de naturalité de la race, sans infléchir la conception naturaliste de l'homme ${ }^{13}$.

Ce présupposé trouve son principe dans la tradition philosophique aristotélicienne, mais il prend la forme qu'on lui connaît dans un moment particulier de l'histoire (au moins « occidentale »). Le "Grand Partage », au XVIle siècle, instaure un schéma binaire de représentation du monde: avant/après, ici/là-bas, nature/culture, etc. Sous l'apparence d'équivalence de deux pôles distincts, le schéma est en fait toujours asymétrique ${ }^{14}$, car il est soutenu par une conception à la fois ethnocentrique et téléologique. De telle sorte que se forment des chaînes d'équivalences morales en même temps que des couples d'oppositions (ici $=$ nous $=$ culture $/$ là-bas $=$ eux $=$ nature $\ldots)$. II en résulte un double présupposé, au fondement même de la définition de la science. D'un côté, une séparation entre " le savant et le politique ", soit entre deux champs conçus comme systèmes autonomes de représentation (représentation des sujets par l'Etat, et des objets par la Science) ${ }^{15}$. D'un autre côté, une distinction entre sciences de la nature (souvent dites " dures ») et sciences du social. Notre problème vient donc en partie du fait que la notion de " race " se situe à l'articulation de ces univers. Les "races" ont en effet été élaborées au XIXe siècle par emprunts et par nouage entre ces univers, générant un hybride de sciences socialesnaturelles (l'anthropologie physique), et un hybride scientifico-politique (la catégorie de « race »).

De ce fait, la critique de la confusion entre nature et social n'a pas nécessairement clarifié leur rapport. La catégorie de nature hérite de surcroît d'un statut ambivalent, dans l'asymétrie du partage. Ceci, pour deux raisons. D'une part, si elle est objet des sciences (naturelles), elle est aussi celui de la (bio-)politique ${ }^{16}$. D'autre part, la charge antiraciste

\footnotetext{
12 LORCERIE F., "Différences culturelles, confrontations identitaires et universalisme : questions autour de l'éducation interculturelle », Carrefours de l'éducation, 2002/2, n¹4.

${ }^{13}$ On retrouve ce problème dans la catégorie de sexe. En effet, la distinction sexe/genre n'a pas résolu la tension, n'ayant qu'extrait de la « nature » certaines dimensions rattachées à l'imaginaire social du sexe. Une mise en relation des catégories de sexe et de race mériterait d'être réfléchie, a fortiori car leur mise en équivalence donne parfois lieu à un étonnant raisonnement : le problème de la race est fréquemment tenu pour plus sensible que celui du sexe, en même temps que le sexe est tenu pour plus naturel que la race...

14 LATOUR B., Nous n'avons jamais été modernes. Essai d'anthropologie symétrique, Paris, La Découverte, Poche, 1997.

15 SHAPIN S., SCHAFFER S., Le Léviathan et la pompe à air. Hobbes et Boyle entre science et politique, Paris, La Découverte, 1993.

${ }^{16}$ FOUCAULT M., Il faut défendre la société. Cours au collège de France de 1976, Paris, Gallimard/Seuil, 1997.
} 
contre l'idée d'une pluralité de natures, au nom de l'unité du "genre humain », a eu pour effet de renvoyer l'idée de pluralité sur le terrain de la culture. Outre le fait que cela a compliqué la question de la culture, cela n'a pas changé la conception de la nature ni résolu la question de son statut. La nature reste en fait indiscutable ${ }^{17}$. C'est ce qui explique, à mon sens, le problème que pose la persistance de cette idée de "race », laquelle constitue comme un passage organisé entre des champs que l'on voudrait tenir séparés.

\section{Une asymétrie dans le raisonnement : le statut de la « nature »}

Face au culturalisme, le front de la critique cognitiviste s'est approfondi et/ou déplacé, en particulier dans le renouvellement des théories de l'ethnicité. Depuis les années 1960, et les travaux de l'anthropologue Fredrik Barth, s'est diffusée une approche constructiviste des groupes ethniques. Elle propose de saisir ces formations sociales à partir d'une problématique de la frontière ${ }^{18}$. Ceci a permis de mettre en lumière le fait que ces groupes se constituent à partir de situations d'altérité, les frontières étant élaborées à travers une opposition et des interactions entre "Nous " et "Eux ». Cette approche conduit à historiciser les statuts ethniques et à mettre en évidence le caractère variable des marques et des signes de reconnaissance et d'identification des groupes. Ce renversement de perspective, par rapport à une ethnologie objectiviste, se diffuse dans le contexte scientifique français - même si cela reste très progressif et limité.

Bien qu'une telle approche de l'ethnicité soit également appliquée à l'idée de « race », il faut remarquer que l'on continue pourtant de traiter différemment les deux notions, sans que cela ne se justifie uniquement par un référent distinct (nature/culture). Par exemple, si des auteurs ont construit un équivalent de racisme dans la notion d'ethnisme ${ }^{19}$, celle-ci porte sur la dimension idéelle, et n'est que partiellement transférée aux pratiques. Ainsi, l'on parle en même temps de "discrimination raciste » (en récusant le qualificatif " racial » comme supposant l'ordre raciste) et de " discrimination ethnique » (qui, pour le coup, ne serait pas " ethniste »). On peut donc voir dans le choix terminologique distinct entre "race " et " ethnie » le maintien d'une étonnante asymétrie. Tout se passe comme si l'on entérinait et prolongeait une différence de nature entre les catégories d'« ethnie » et de "race » différenciation qui semble en outre discutable au regard de l'histoire de ces termes dans la littérature rac(ial)iste.

Pourquoi cette asymétrie ? La validité différente implicitement prêtée à « race " et " ethnie » semble découler d'une reprise en compte par les sciences sociales de la "culture " (à

17 LATOUR B., Politiques de la nature. Comment faire entrer les sciences en démocratie ?, Paris, éd. La Découverte, 1999.

18 BARTH F., "Les groupes ethniques et leurs frontières », in Poutignat P., Streiff-fénart J. Les théories de l'ethnicité, Paris, PUF, 1995.

19 DE RUDDER V., POIRET C., VOURC'H F., L'inégalité raciste. L'universalité républicaine à l'épreuve, Paris, PUF, 2000. 
condition de sa dés-essentialisation) en même temps que le maintien à distance de la notion de « nature » - toujours référée à la biologie sans être dé-naturalisée ${ }^{20}$. La séparation du «Grand Partage » se retrouve ainsi prolongée sous la forme d'une asymétrie de traitement, liée au fait que l'on continue d'investir la nature de qualités fixatives et unitaires. Comme le dit Bruno Latour l'investissement des cultures se fait «sur fond de nature unifiée $»^{21}$. La remarque peut être rapprochée de celle que fait Judith Butler à propos du genre: "la relation entre culture et nature présupposée par certains modèles de "construction" du genre implique une culture ou une puissance du social qui agirait sur la nature, elle-même considérée comme une surface passive, en dehors du social et en constituant pourtant la contrepartie nécessaire ${ }^{22}{ }^{22}$

La sociologie, tenant la race pour un rapport social, peut être tentée d'évacuer la question de la physicalité chère aux théories racistes, en tant que surface d'inscription de la " nature ». II s'agit bien entendu d'échapper au fait que traiter la part physique/physiologique de cette question comme un donné " présuppose et consolide les conditions normatives de sa propre émergence $\nu^{23}$. Mais on peut symétriquement se demander pourquoi il faudrait considérer la nature comme un donné ? En rejetant l'idée de «natures humaines différentes " («les races ») pour observer l'usage de cette conception dans les rapports de pouvoir (la race), le débat reste borné par un refus de l'hypothèse d'une multiplicité (vs une unicité) de nature(s). II s'agit bien entendu de se garder d'adopter la perspective raciste. Or, c'est sur ce point qu'un déplacement mériterait d'être envisagé. Car la logique raciste me semble d'abord tenir à la croyance dans la fixité de la nature, le principe de la différence n'intervenant qu'en second. La difficulté rencontrée est donc liée à l'héritage par la science de cette même croyance issue du "Grand Partage ». Sans prendre ici parti dans le débat, je voudrais au moins prendre celui $d u$ débat, auquel invitent certains auteurs, à partir d'une redéfinition de la « nature » en tant qu'agencement et " jeu de contraste ${ }^{24}$. J'entends toutefois que ces propositions heurtent de plein fouet une approche privilégiant la catégorisation ${ }^{25}$. Un considérable travail de réélaboration serait donc nécessaire.

\section{La « purification » ou l'ambivalence d'un idéal de nature}

La thématique "des races », chez les auteurs racistes, est traversée et ordonnée par la hantise de "l'impureté ». "Les races » étant tenues pour des catégories soit idéalement soit originellement pures - du moins certaines d'entre elles - ces auteurs déplorent un « mélange

\footnotetext{
${ }^{20}$ Malgré des travaux cognitivistes dans ce domaine, comme ceux de Francisco Varela.

${ }^{21}$ LATOUR B., La fabrique du droit. Ethnographie du Conseil d'Etat, Paris, La Découverte/Poche, p.264.

22 BUTLER J., Ces corps qui comptent. De la matérialité et des limites discursives du " sexe », Paris, éd. Amsterdam, 2009, p.18.

23 Ibid, p.23.

${ }^{24}$ LATOUR B., La fabrique du droit, op. cit., p.265.

25 «Entretien avec Bruno Latour », Tracés, n¹0, hiver 2006.
} 
des sangs », tantôt présenté comme irrémédiable, tantôt justifiant une réaction eugéniste. La question de la pureté n'est toutefois pas l'apanage du schéma raciste. D'une part, le tabou qui entoure aujourd'hui le terme de "race " peut être vu comme la trace d'une impureté prêtée à la catégorie elle-même. Cela résulte d'un retournement de la croyance raciste dans cette catégorie. Ce retournement dérive de l'activité politico-scientifique d'ordre moral ayant conduit à retirer à la « race " sa pertinence comme réalité attestable et comme taxonomie scientifiquement valide. D'autre part, et c'est un paradoxe, la critique du syncrétisme se fait au nom de ce que le raisonnement "mélange » des registres que les sciences distinguent. Le maintien des divisions du travail de la connaissance recourt luiaussi à un processus de purification. La critique relève donc, d'une certaine façon, d'un procès en impureté scientifique du raisonnement. C'est au nom d'un idéal scientifique de la pureté que l'on voudrait se débarrasser d'un terme socialement et scientifiquement impropre. Dans ce débat, deux schémas de purification s'opposent... mais s'allient aussi historiquement. On a cru, dans les années 1960, qu'il suffisait d'aller au terme d'un processus de purification, en renvoyant "les races" au rebut de l'histoire des pseudoconcepts. Mais, outre que cette position n'a pas dissout les catégories raciales, elle se fait au risque d'effacer le motif et la justification du racisme, en récusant le nom de « race ${ }^{26}$.

Le problème est donc à double fond, si l'on accepte l'idée que la contribution des sciences à l'élaboration des catégories raciales, aux XIXe et XXe siècles, n'est pas uniquement le fruit de leur instrumentalisation idéologique. Cela tient aussi à un processus interne à la science, qui est celui d'une élaboration du savoir reposant sur un principe de purification. Ce processus traverse par exemple la question du classement. L'anthropologie physique a été la passerelle entre les deux univers, en poussant le raisonnement quant à la purification catégorielle du point de vue de la nature humaine. Je ne prétends pas ici que la science fonctionne sur le même schéma que l'idéologie. Une interrogation sur le lien entre deux usages de la notion de "pur " semble néanmoins s'imposer. Bien entendu, les conditions de légitimation du discours ne sont pas identiques, entre les usages théorique et banal des catégories de «race ${ }^{27}$. Par ailleurs, le sens de la «purification » n'est pas simplement transposable du régime idéologique au régime scientifique. Mais la liaison entre les deux n'est pas uniquement métaphorique. Le "pur » semble constituer, dans les deux cas, un idéal d'ordre référé à une conception définitive de la nature - que cet idéal soit tenu pour premier ou pour dernier.

\footnotetext{
${ }^{26} \mathrm{Or}$, « il n'est pas soutenable de prétendre qu'une catégorie qui organise des Etats (...), qui entre dans la Loi n'existe pas. (...) Non, la race n'existe pas. Si, la race existe. Non certes, elle n'est pas ce qu'on dit qu'elle est, mais elle est néanmoins la plus tangible, réelle, brutale des réalités. " GUILLAUMIN C., " "Je sais bien, mais quand même" ou les avatars de la notion "race" ", Le genre humain, Paris, Fayard, 1981, p.65.

${ }^{27}$ GUILLAUMIN C., « Usages théoriques et usages banals du terme race », Mots, vol. 33, n¹, 1992.
} 
A un niveau général, ces questions du «pur » ou du «tabou » travaillent ensemble pour mettre en jeu les frontières et les places des groupes sociaux ${ }^{28}$. Si les principes de tri peuvent différer, il y a des raisons de voir dans certaines catégories - celle de "races ", mais aussi celle de "communautés ${ }^{29}$ - des passerelles organisant des passages du champ politique au champ scientifique et réciproquement. L'équivalence s'établirait dans le passage d'une catégorie à l'autre, ou autrement dit dans le report de certaines questions vers d'autres. Le passage de "race » à « ethnie " apparaîtrait ici comme un indice de ce mécanisme de report. Un autre indice serait à rechercher dans le statut que la sociologie accorde à son objet, le « social ». Celui-ci est fréquemment et paradoxalement aussi référé à un principe de pureté. En utilisant le terme de " social " comme qualificatif (" faits sociaux ", etc.), on en vient en effet souvent moins à traiter du caractère relationnel d'un phénomène ${ }^{30}$, qu'à suggérer une nature spécifique de celui-ci. Comme si social était le nom d'une " seconde nature ${ }^{31}$. Certains auteurs vont ainsi jusqu'à attribuer à la « race " «le caractère de pure construction sociale $»^{32}$, justifiant ainsi les guillemets. De ce point de vue, la catégorie de " race " poserait le problème d'être scientifiquement impurifiable, à moins de la réinvestir dans le seul registre social (la biologie tendant à la récuser). Ce report dans un autre registre n'efface cependant pas le problème (en témoignent les guillemets), car s'y entremêlent irrémédiablement des préoccupations morales, des soubassements idéologiques, des positions politiques, etc.

\section{Le transfert de la nature dans le social et le risque de la réification}

Si les nouvelles théories de l'ethnicité bénéficient d'une formidable relativité - à l'instar des travaux sur le genre, en quelle mesure applique-t-on ce relativisme à la catégorie du « social » ? On peut voir ici le prolongement d'un défaut de symétrie dans le raisonnement. Dans le discours sociologique apparaît fréquemment une opposition entre d'une part, les catégorisations "ethnico-raciales " des groupes, et d'autre part, des "caractéristiques objectives de leur milieu familial et social $»^{33}$. Véronique De Rudder soutient que «la catégorisation ethnique ou raciale n'a pas le même type de fonctionnement matériel ou objectif que les autres grandes variables descriptives (âge, sexe, catégorie socioprofessionnelle, logement...) qui, elles-mêmes, ont des modes de construction

\footnotetext{
${ }^{28}$ DOUGLAS M., De la souillure. Essai sur les notions de pollution et de tabou, Paris, La Découverte/Poche, 2001.

${ }^{29}$ DHUME F., « Commun, communauté(s), "communautarisme”. Les frontières de la socia(bi)lité », Mana, $n^{\circ} 16$.

${ }^{30} \mathrm{Au}$ sens que donne Georg Simmel à ce terme; la sociologie étant alors pensée dans une approche relationniste. SIMMEL G., Sociologie. Etudes sur les formes de la socialisation, Paris, PUF, 1999.

${ }^{31}$ LATOUR B., Changer de société, refaire de la sociologie, Paris, La Découverte, 2006

32 POIRET C., «Articuler les rapports de sexe, de classe et de "race". Quelques enseignements du débat nordaméricain ", Revue européenne des migrations internationales, vol.21, $n^{\circ} 1,2005$, note $n^{\circ} 4$. J'entends ici la part de métaphore, mais cela mériterait justement discussion.

${ }^{33}$ VALLET L.-A., " Les élèves étrangers ou issus de l'immigration: les résultats du panel français dans une perspective comparative ", in Aubert F., Tripier M., Vourc'h F., Jeunes issus de l'immigration : de l'école à l'emploi, Paris, Ciemi/L'Harmattan, 1997.
} 
hétérogènes. ${ }^{34}$ S'il faut bien sûr entendre que les catégories se différencient dans leur fonctionnement, comment se distribue leur « objectivité » ? N'y a-t-il pas là, implicitement, un raisonnement qui naturalise le social, en lui conférant une objectivité tout en renvoyant « l'ethnico-racial » à son opposé ? On fait alors comme si ces catégories étaient d'essences intrinsèquement différentes. Dominique Schnapper va plus loin encore, évoquant des "groupes définis par des critères économiques et sociaux universels ", pour expliquer son refus d'utiliser « des critères ethniques $»^{35}$.

Que chaque catégorie soit construite différemment, et pose en conséquence des questions quant à sa mise en équivalence avec d'autres, me semble être un défi majeur de la réflexion épistémologique. Mais ce n'est pas de cela dont parlent ces critiques de l'usage de la catégorie ethnico-raciale, lorsqu'elles dénient la naturalité et la pertinence de la " race », et insistent sur la fragilité de son statut au nom d'une objectivité intrinsèquement supérieure d'autres catégories. Tout se passe comme si une hiérarchie essentielle se maintenait, empêchant une mise en équivalence constructiviste de ces catégories. Le risque de cette position est bien de réifier l'objet et l'approche qui fondent l'interrogation sociologique : le social. On peut entendre la prudence qu'il y a derrière la distinction entre les catégories. On peut également être sensible aux présupposés éthiques qui sous-tendent ces positions de mise à distance critique de l'« ethnico-racial ». Mais alors c'est peut-être (aussi) de cela dont il nous faut parler : de l'hybridité nécessaire de ces positions.

\section{La race comme un problème : I'intrication de la science, de la morale et de la politique}

En manipulant de telles notions, nous ne pouvons nous départir d'interrogations normatives, avec l'inquiétude que génère l'expérience du désastre qui est aujourd'hui nôtre. Nous sommes responsables de ce que nous faisons d'un héritage bien difficile à assumer : tout ce qui a été fait au nom de la race. C'est irréductiblement une responsabilité morale et politique. Et pourtant, le débat scientifique peut parfois se dérouler comme si l'on n'était qu'en face de positions scientifiquement justifiées; comme si dans les choix opérés au nom de la recherche, l'éthique et la morale n'étaient pas co-déterminantes, etc. Catégorie-limite et catégorie-passerelle, la « race » est un point voué à l'instabilité. Sauf à recourir à l'imaginaire raciste, elle n'est jamais entièrement purifiable, de même qu'elle n'est jamais définitivement évacuable, ni isolable, ni attribuable. Nous avons affaire à une question irréductiblement en réseau, et en tension. La catégorie rend ainsi compte, à sa façon, de l'aporie d'une logique qui serait celle de la purification totale, y compris dans le registre scientifique. Pourtant, cette dimension problématique me semble trop rarement être au centre de la réflexion.

\footnotetext{
${ }^{34}$ DE RUDDER V., «Quelques problèmes épistémologiques liés aux définitions des populations immigrantes et de leur descendance », in Aubert F., Tripier M., Vourc'h F. op. cit., p.27.

35 SCHNAPPER D., intervention au séminaire national: «Lutter contre les discriminations: la diversité à l'Ecole », ministère de l'Education nationale, Paris, lycée Louis-Legrand, 7 octobre 2009.
} 
L'évacuation du débat sur la nature, que ce soit la physicalité du social ou l'hypothèse de la pluralité de la nature, me semble poser trois problèmes. D'abord, cette évacuation a pour effet de limiter la question constructiviste, en écartant implicitement de son domaine de réflexion ce présupposé fort problématique d'un «fond de nature ». Ensuite, paradoxalement, cela revient à conférer au social une essence propre, comme une "seconde nature ". La sociologie risque ici de réifier son objet. Enfin, en se refusant à assumer ce que cette question engage comme problèmes, nous nous privons dans le même mouvement de voir ce qu'elle engage comme potentiel de conflictualité. Or, c'est à ce point précis du débat, me semble-t-il, que plusieurs formes contemporaines de lutte prennent le plus d'intensité. A savoir, autour des catégories ethnico-raciales ou de genre. Défendre une telle approche constructiviste ne doit justement pas s'éteindre dans un "constructionisme vulgaire $\|^{36}$ qui, considérant que toutes les catégories sociales sont construites, balaierait d'un geste la pertinence de leur caractère de ressource pour les luttes des minorisés ${ }^{37}$. L'approche constructiviste n'a pas à substituer à un surplomb essentialiste un abîme relativiste. II s'agit au contraire d'accepter ces catégories dans leur paradoxe, et de voir que ce paradoxe constitue aussi un front possible de résistance aux effets de pouvoir catégoriels.

Pour rendre compte de ces tensions, on peut tenir la race comme le nom d'un problème. Problème, dans le sens que lui donnait Abdelmalek Sayad à propos de " l'immigration ${ }^{38}$, à savoir, un nœud irrémédiable entre objet sociologique et objet politique. La " chose » étant impurifiable, il n'est pas sûr que nous trouvions un autre compromis que celui que propose par exemple le droit, aux prises avec cette insoluble contradiction: faire exister pour révoquer ${ }^{39}$, nommer pour invalider. La race fait problème, pour un projet scientifique qui rêverait de l'expurger de toute ambiguïté, à défaut de pouvoir s'en débarrasser. Faire des sciences sociales dans l'impureté du monde social, n'est-ce pas finalement là l'horizon que nous pouvons pragmatiquement assumer?

Fabrice DHUME, ISCRA

Novembre 2009 - Février 2010

\footnotetext{
36 CRENSHAW K., " Mapping the margins. Intersectionnality, identity politics, and violence against women of color », in Albertson Fineman M., Mykitiuk R., (éds), The public nature of private violence, New York, Routledge, 1994.

37 Selon les théories de l'ethnicité, le groupe qui impose sa norme comme étant l'ordre « normal » ou « neutre » est dit majoritaire. Les autres groupes sont en conséquence dit minoritaires. En parlant de minorisés, j'insiste sur l'asymétrie de ces statuts et la primauté de l'imputation par le « dominant » de statuts aux uns et aux autres.

38 SAYAD A., L'immigration ou les paradoxes de l'altérité, Paris, Raisons d'agir, 2006.

${ }^{39}$ Cf. les remarques de : BUTLER J., Le pouvoir des mots. Politique du performatif, Paris, éd. Amsterdam, 2004.
} 